\title{
Both taurine and albumin support mouse sperm motility and fertilizing ability in vitro but there is no obligatory requirement for taurine
}

\author{
Lynn R. Fraser \\ Department of Anatomy and Human Biology, King's College London, Strand, London WC2R 2LS, \\ U.K.
}

\begin{abstract}
Summary. When mouse spermatozoa were washed immediately upon release from the epididymis, preincubated for up to $120 \mathrm{~min}$ in PVA-containing, albumin-free medium and assessed for their ability to fertilize cumulus-intact eggs in vitro, they were poorly fertile in comparison with their unwashed counterparts in the same medium. Fertilizing ability could be significantly improved by introducing taurine or albumin or by washing a second time at the end of preincubation. The most effective treatment was provided by the continuous presence of low concentrations $(0.05-0.1 \mathrm{mg} / \mathrm{ml})$ of BSA, similar to the amount of albumin detected in the supernatants removed during washing. There was no evidence that acrosome loss was inhibited by washing; rather, it was enhanced by the removal of a surface component which inhibits the acrosome reaction. The presence of taurine did not further increase this response. Motility, reduced in washed suspensions, was improved by the presence of taurine or albumin and experimental results suggest that this was a major factor in the improvement of fertilizing ability after introduction of these compounds. Although taurine, hypotaurine and albumin were all found in the sperm washings and thus would be present in unwashed, fertile samples, low concentrations of albumin were able to maintain full fertilizing ability. Therefore, unlike hamster spermatozoa, mouse spermatozoa would not appear to have an obligatory requirement for a motility stimulating factor such as taurine.
\end{abstract}

\section{Introduction}

Sperm capacitation requires a species-dependent interval during which changes that promote the spermatozoon's ability to fertilize an egg occur (Bedford, 1970). Conditions that support capacitation in vitro have been examined for a variety of laboratory species and it has become evident that there are certain molecules that must be present or that substantially enhance sperm fertilizing potential (reviewed by Fraser, 1984a). For example, albumin is specifically able to trigger the acrosome reaction in capacitated spermatozoa and, in its absence, the acrosome reaction is inhibited (Lui \& Meizel, 1977; Fraser, 1985). Albumin is also effective in maintaining sperm motility during incubation in vitro (e.g. Suter, Chow \& Martin, 1979; Harrison, Dott \& Foster, 1982; Fraser, 1985). Some spermatozoa appear to require further molecules, in addition to albumin, to maintain adequate motility and fertility. For example, hamster spermatozoa require a 'sperm motility factor' which has been tentatively identified as either taurine or hypotaurine, sulphur-containing $\beta$-amino acids (Mrsny, Waxman \& Meizel, 1979; Meizel, Lui, Working \& Mrsny, 1980; Leibfried \& Bavister, 1981). There is also some evidence that these amino acids may help stimulate the acrosome reaction in hamster spermatozoa.

The present study was designed to examine the effects of introducing taurine and bovine serum albumin (BSA) to epididymal mouse spermatozoa washed shortly after release to remove 
epididymal fluid components; motility, acrosome loss and fertilizing ability were assessed after preincubation of spermatozoa for up to $120 \mathrm{~min}$.

\section{Materials and Methods}

Media. A modified Tyrode's solution (Fraser, 1983) was used throughout. The macromolecular component was either albumin (crystalline BSA at $4 \mathrm{mg} / \mathrm{ml}$; Sigma, Poole, Dorset, U.K.) or polyvinyl alcohol (PVA) at $1 \mathrm{mg} / \mathrm{ml}$. PVA is a macromolecule which supports sperm motility but not the acrosome reaction (Bavister, 1981; Fraser, 1985). In some experiments the amino acids taurine and hypotaurine (Calbiochem-Behring, La Jolla, CA, U.S.A.) were used.

In-vitro fertilization. Mature female TO mice ( $>8$ weeks) were induced to superovulate with i.p. injections of 7.5 i.u. PMSG (Gestyl: Organon, Morden, Surrey, U.K.) and, after 54 h, 5 i.u. hCG (Pregnyl: Organon). Eggs were recovered $14 \mathrm{~h}$ after hCG injection by releasing cumulus clots from the oviducts directly into sperm suspensions. When neccessary hCG was injected asynchronously to ensure that aged eggs would not be used.

Sperm suspensions were prepared by releasing the contents of 1 cauda epididymidis from each of 2 mature ( $>8$ weeks) male TO mice into $1 \mathrm{ml}$ medium; thus, 2 suspensions could be prepared from each pair of males. Suspensions were preincubated for a total of $120 \mathrm{~min}$ at $37^{\circ} \mathrm{C}$, with fertilizing ability being assessed after preincubation for 30 and/or $120 \mathrm{~min}$. Aliquants were diluted $\sim 10$-fold to a final concentration of $1-2 \times 10^{6}$ spermatozoa $/ \mathrm{ml}$ and eggs were released into $300 \mu 1$ droplets of diluted suspension.

Incubations were carried out in $30 \mathrm{~mm}$ plastic culture dishes (Sterilin, Teddington, Middlesex, U.K.) and medium was overlaid with autoclaved liquid paraffin (Boots, Nottingham, U.K.). The gas phase was $5 \% \mathrm{CO}_{2}-5 \% \mathrm{O}_{2}-90 \% \mathrm{~N}_{2}$.

Washing of sperm suspensions. One volume of sperm suspension was gently mixed with 2 volumes of the medium used for sperm release and centrifuged at $750 \mathrm{~g}$ (room temperature) for $5 \mathrm{~min}$. After aspiration of the supernatant, the pellet was resuspended to the original volume in the appropriate medium.

Routine assessments. Eggs were transferred from sperm suspensions to droplets of fresh medium $65 \mathrm{~min}$ after mixing of gametes and fixed at $75 \mathrm{~min}$ by addition of excess neutral buffered formalin ( $4 \%$ formaldehyde). A brief exposure to hyaluronidase before fixation was required to complete removal of cumulus cells from eggs incubated with washed suspensions (see Fraser, 1984b). Eggs were stained and mounted (Fraser, 1983), then assessed for fertilization. They were considered to be fertilized if they contained a decondensed sperm head and had resumed the second meiotic division. The proportion of eggs at telophase or beyond of meiosis II which possessed a fully decondensed sperm head, a measure of the rapidity of sperm penetration, was calculated.

Preincubated, diluted sperm suspensions were assessed both for proportion of motile cells and expression of hyperactivated motility.

Acrosome loss after different treatments was assessed by filtering sperm suspensions to select motile cells. Suspensions were applied to short columns of Sephadex G-25 prepared in Pasteur pipettes (Fraser, 1985) and eluted with the appropriate medium; approximately $90 \%$ of eluted cells were motile. After fixation in formalin, slides were prepared (Fraser, 1983) and at least 100 cells were evaluated in each sample for the presence or absence of the acrosome.

Statistical analysis was performed using Cochran's test for the combination of $2 \times 2$ contingency tables (Snedecor \& Cochran, 1967).

Amino acid analysis of sperm washings. Sperm suspensions were prepared in macromolecule-free medium (both epididymides from 1 male into $1 \mathrm{ml}$ ) and centrifuged to pellet the spermatozoa. The cell-free supernatant was deproteinized by adding $15 \mathrm{mg}$ sulphosalicylic acid to each $1 \mathrm{ml}$ of supernatant and centrifuging $(11600 \mathrm{~g})$. The deproteinized samples were applied to a Locarte 
amino acid analyser and eluted using lithium salts and a 4 buffer system; the $23 \mathrm{~cm}$ column was packed with sulphonated polystyrene resin. In addition to supernatant samples, some of the sperm pellets were resuspended in $1 \mathrm{ml}$ medium, frozen-thawed to break up the cells, deproteinized and analysed. Finally, medium containing $4 \mathrm{mg} \mathrm{BSA} / \mathrm{ml}$ was deproteinized and analysed for any amino-acid contaminant. Values were calculated from measurements made with fine calipers.

\section{Results}

Series I: the effect of washing sperm suspensions in BSA-free medium

Sperm suspensions were prepared in BSA-containing and PVA-containing media. At 5 min after sperm release, aliquants of each suspension were washed, resuspended in medium of the same composition and preincubated further. At 30 and 120 min after release, washed and unwashed suspensions were diluted and eggs were added to assess fertilizing ability. Six replicate experiments were performed $(\mathrm{N}=6)$.

Unwashed, BSA-containing suspensions showed a typical pattern of fertilizing ability, with relatively few eggs fertilized after sperm preincubation for $30 \mathrm{~min}$ and the great majority fertilized

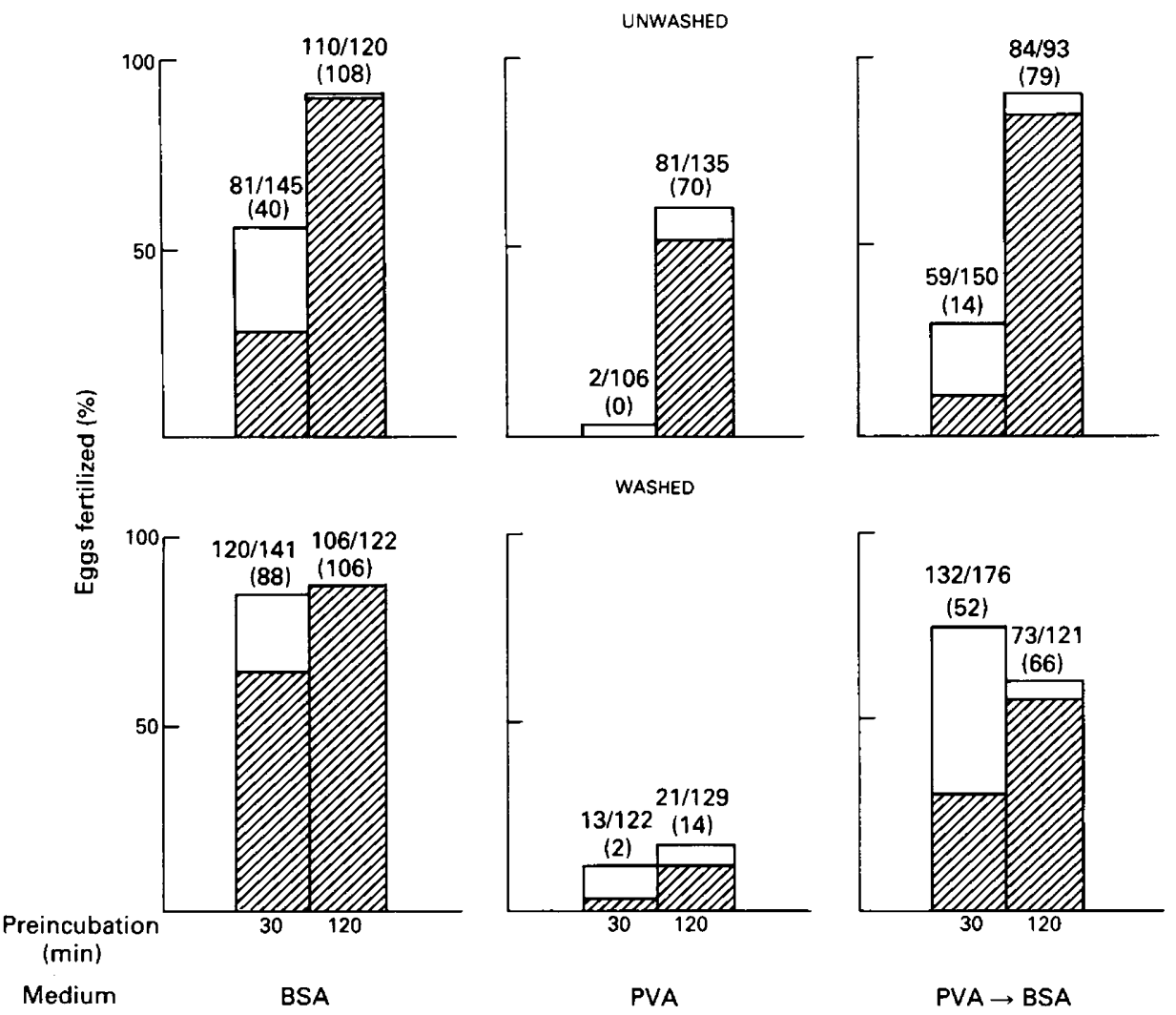

Fig. 1. In-vitro fertilizing ability of unwashed and washed (at $5 \mathrm{~min}$ ) mouse sperm suspensions preincubated in PVA- or BSA-containing medium. The bars represent $\%$ of eggs possessing a fertilizing sperm head and the hatched areas represent $\%$ of eggs with fully decondensed sperm heads. The figures above each bar indicate the number of eggs fertilized/total eggs and the figures in parentheses indicate the number of eggs with fully decondensed sperm heads. 
after $120 \mathrm{~min}$ (Fig. 1). Consistent with earlier studies (e.g. Fraser, 1984b, 1985) rapid synchronous penetration of fertilizing spermatozoa was observed in the samples preincubated for $120 \mathrm{~min}$ but not those at $30 \mathrm{~min}$. In contrast to the $30 \mathrm{~min}$ unwashed suspensions, significantly $(P<0.05)$ more eggs were fertilized by the $30 \mathrm{~min}$ washed counterparts and penetration was rapid; this high level of fertility was maintained during preincubation for $120 \mathrm{~min}$.

Unwashed suspensions preincubated in PVA-containing medium were poorly fertile after $30 \mathrm{~min}$ and quite fertile after $120 \mathrm{~min}$ preincubation (Fig. 1), while the comparable washed suspensions were very poorly fertile at both times $(P<0.01$ compared with 120 min unwashed PVA spermatozoa). Fertilizing ability of all these suspensions could be improved by introducing BSA at the time of dilution, i.e. immediately before egg addition. In particular the fertilizing ability of the washed PVA suspensions was significantly enhanced $(P<0.01)$ by the addition of BSA, although the washed spermatozoa preincubated for $120 \mathrm{~min}$ were still less fertile than their unwashed counterparts $(P<0.05)$. The following experimental series were designed to examine further the poor performance of the washed PVA-containing suspensions.

\section{Series II: washing BSA-free sperm suspensions twice improves fertilizing ability}

Sperm suspensions were again prepared in BSA- and PVA-containing media. After 5 min, an aliquant of the PVA suspension was washed and then all samples were preincubated for a total of 120 min. At this time, an aliquant of the unwashed suspension was washed and resuspended in PVA-containing medium, while an aliquant of the sample washed at $5 \mathrm{~min}$ was subjected to a second washing. The PVA samples were diluted into both PVA- and BSA-containing media for fertility assessment $(N=3)$.

The unwashed BSA suspensions were highly fertile, with $91 \%$ of eggs $(61 / 67)$ fertilized and all with fully decondensed sperm heads. Results obtained with the PVA suspensions are presented in Fig. 2. Samples washed once, after 120 min preincubation, and their unwashed counterparts were quite fertile, with $75-85 \%$ of eggs fertilized. As in Series I, samples washed at $5 \mathrm{~min}$ were
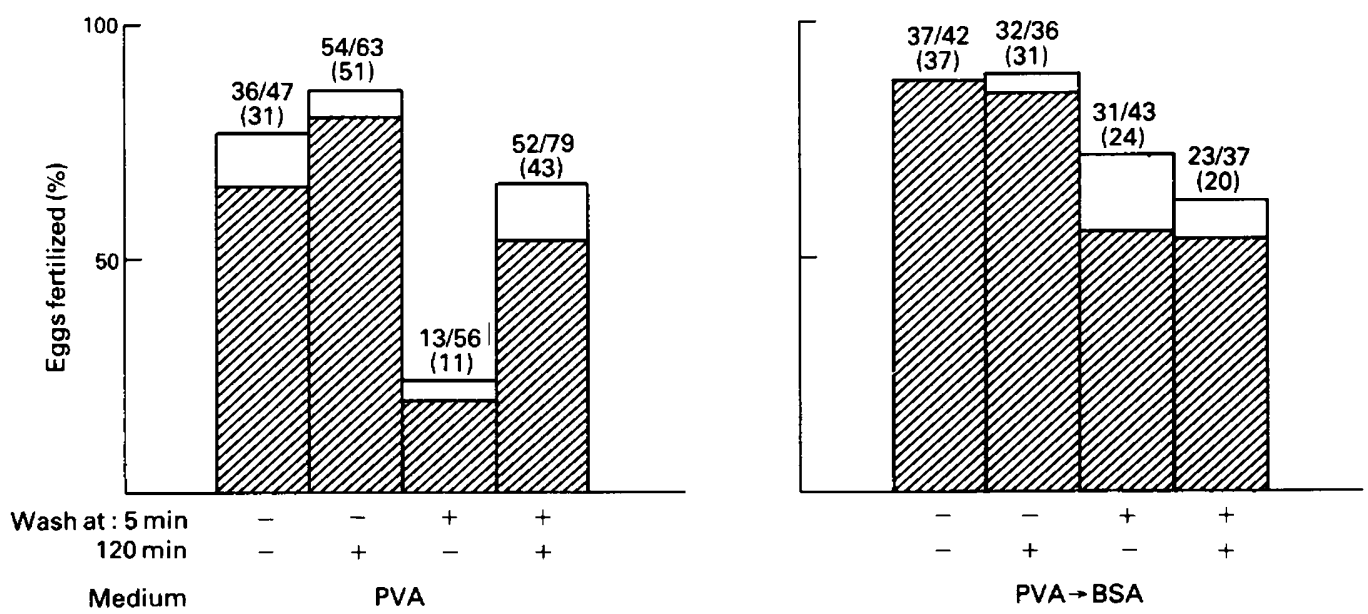

Fig. 2. In-vitro fertilizing ability of unwashed and washed (at 5 and/or $120 \mathrm{~min}$ ) mouse sperm suspensions preincubated in PVA-containing medium. Each suspension was assessed for fertilizing ability in the presence of PVA and BSA. The bars represent $\%$ of eggs possessing a fertilizing sperm head and the hatched areas represent $\%$ of eggs with fully decondensed sperm heads. The figures above each bar indicate the number of eggs fertilized/total eggs and the figures in parentheses indicate the number of eggs with fully decondensed sperm heads. 
significantly less fertile $(P<0.05)$ than unwashed spermatozoa but this difference was removed by washing a second time at $120 \mathrm{~min}$. The introduction of BSA also enhanced fertilizing ability of the 5 min washed spermatozoa significantly $(P<0 \cdot 05)$.

Series III: what is the minimum concentration of BSA that will maintain high fertility in washed sperm suspensions?

The first two series of experiments indicated that the introduction of BSA at $4 \mathrm{mg} / \mathrm{ml}$ to washed, preincubated suspensions improved fertilizing ability. In the third series, suspensions released into PVA-containing medium were washed at $5 \mathrm{~min}$, then resuspended and preincubated for $120 \mathrm{~min}$ in media with very low concentrations of BSA: $0 \cdot 01,0 \cdot 05$ or $0 \cdot 1 \mathrm{mg} / \mathrm{ml}(\mathrm{N}=4)$.

Unwashed suspensions preincubated in medium containing $4 \mathrm{mg} \mathrm{BSA} / \mathrm{ml}$ were highly fertile (Table 1), while the washed PVA suspensions were significantly less fertile $(P<0.01)$. The inclusion of $0.01 \mathrm{mg} \mathrm{BSA} / \mathrm{ml}$ did not improve fertility, but both 0.05 and $0.1 \mathrm{mg} \mathrm{BSA} / \mathrm{ml}$ supported fertilization rates that did not differ significantly from the $4 \mathrm{mg} \mathrm{BSA} / \mathrm{ml}$ control group.

Table 1. Critical concentration of BSA required to maintain fertilizing ability of washed mouse sperm suspensions

\begin{tabular}{|c|c|c|c|c|c|c|}
\hline \multicolumn{2}{|c|}{ Medium } & \multirow[b]{2}{*}{$\begin{array}{c}\text { Wash at } \\
5 \mathrm{~min}\end{array}$} & \multicolumn{3}{|c|}{ Eggs fertilized } & \multirow[b]{2}{*}{$\begin{array}{l}\text { Maximal nuclear } \\
\text { development } \dagger, \%\end{array}$} \\
\hline Release & $\begin{array}{l}\text { Preincubation } \\
+ \text { fertilization }\end{array}$ & & No. & $\%$ & (range) & \\
\hline $4 \mathrm{mg} \mathrm{BSA} / \mathrm{ml}$ & $4 \mathrm{mg} \mathrm{BSA} / \mathrm{ml}$ & - & $109 / 121$ & $90 \cdot 1$ & $(67-100)$ & $99 \cdot 1$ \\
\hline I mg PVA $/ \mathrm{ml}$ & $\begin{array}{c}1 \mathrm{mg} \mathrm{PVA} / \mathrm{ml} \\
0.01 \mathrm{mg} \mathrm{BSA} / \mathrm{ml} \\
0.05 \mathrm{mg} \mathrm{BSA} / \mathrm{ml} \\
0.1 \mathrm{mg} \mathrm{BSA} / \mathrm{ml}\end{array}$ & $\begin{array}{l}+ \\
+ \\
+ \\
+\end{array}$ & $\begin{array}{r}54 / 150 \\
44 / 147 \\
81 / 106 \\
106 / 122\end{array}$ & $\begin{array}{l}41 \cdot 4 \\
29 \cdot 9 \\
76 \cdot 4 \\
86 \cdot 9\end{array}$ & $\begin{array}{l}(12-72)^{* *} \\
(15-64)^{* * *} \\
(25-100) \\
(77-100)\end{array}$ & $\begin{array}{l}75 \cdot 9 \\
77 \cdot 3 \\
90 \cdot 1 \\
79 \cdot 2\end{array}$ \\
\hline
\end{tabular}

$\div$ Fertilized eggs at telophase-second polar body with fully decondensed sperm heads.

${ }^{* *} P<0.01,{ }^{* * *} P<0.001$ compared with control group ( $4 \mathrm{mg} \mathrm{BSA} / \mathrm{ml}$ ).

Although the mean percentage of eggs fertilized in the washed PVA suspensions in both this and the following series was low, there was some variability observed among replicates (see range of results in Tables $1 \& 2$ ). While it is possible that this might reflect variation in the albumin contributed by cumulus cell masses, relatively high numbers of eggs/dish (and therefore presumably more albumin) were not consistently correlated with high proportions of fertilized eggs. The most obvious correlation was with sperm motility; the occasional high value was obtained with washed suspensions which had a proportion of motile cells greater than that observed in the majority of suspensions (see Series VI below).

Series IV: taurine enhances the fertilizing ability of washed BSA-free suspensions

In the first set of experiments, the effect of introducing different concentrations of taurine to spermatozoa washed at $5 \mathrm{~min}$ in PVA-containing medium was examined. Washed spermatozoa were resuspended in media containing PVA and taurine at $0.1,0.3$ and $1.0 \mathrm{~mm}$, preincubated for $120 \mathrm{~min}$ and assessed after dilution in the same medium, i.e. taurine was present throughout $(\mathrm{N}=2)$. Results were as follows: unwashed PVA suspensions, $38 / 56$ eggs fertilized $(68 \%)$; washed PVA, 6/36 (17\%); washed PVA + 0.1 mm-taurine, 44/79 (56\%); washed PVA + 0.3 mm-taurine, $14 / 60(23 \%)$; washed PVA + 1.0 mM-taurine, $19 / 78(24 \%)$. 
Since taurine appeared to have a positive effect when present continuously, the second set of experiments was designed to examine more carefully whether the time of contact with taurine was critical to obtain this effect; the concentration of $0.1 \mathrm{~mm}$ was used throughout. A single suspension was prepared in PVA-containing medium and aliquants were washed at $5 \mathrm{~min}$; one was resuspended in PVA-and the other in PVA + taurine-containing medium. After preincubation for $120 \mathrm{~min}$, the washed PVA suspension was assessed after dilution into media containing PVA, PVA + taurine and BSA. The washed PVA + taurine suspension was assessed after dilution in media containing PVA and PVA + taurine $(\mathrm{N}=5)$. In 2 of these replicates, the effect of using $0.1 \mathrm{~mm}$-hypotaurine, rather than taurine, during preincubation and fertilization was examined.

Results are presented in Table 2. The washed PVA suspensions were again poorly fertile, with only $35 \%$ of eggs fertilized. The inclusion of taurine at any stage of gamete incubation significantly enhanced $(P<0.05-0.01)$ the fertilizing ability of these washed spermatozoa. Hypotaurine appeared to be equally effective when compared with taurine. Other data (not tabulated) indicated that taurine at concentrations below $0.1 \mathrm{~mm}(0.05-0.02 \mathrm{mM})$ and in combination with hypotaurine was also effective in improving fertility.

Table 2. Fertilizing ability of mouse sperm suspensions released into PVA-containing medium, washed at $5 \mathrm{~min}$, resuspended in various media and preincubated for $120 \mathrm{~min}$ before assessment

\begin{tabular}{|c|c|c|c|c|c|}
\hline \multicolumn{2}{|c|}{ Medium $\dagger$} & \multicolumn{3}{|c|}{ Eggs fertilized } & \multirow{2}{*}{$\begin{array}{l}\text { Maximal nuclear } \\
\text { development, \% }\end{array}$} \\
\hline Preincubation & Fertilization & No. & $\%$ & (range) & \\
\hline $\mathrm{BSA}_{+}^{+}$ & BSA & $108 / 120$ & $90 \cdot 0$ & $(75-100)$ & $99 \cdot 1$ \\
\hline PVA & $\begin{array}{l}\text { PVA } \\
\text { PVA + T } \\
\text { BSA }\end{array}$ & $\begin{array}{l}53 / 152 \\
71 / 105 \\
53 / 86\end{array}$ & $\begin{array}{l}34.9 \\
67.6 \\
61.6\end{array}$ & $\begin{array}{l}(15-72) \\
(47-76)^{*} \\
(37-100)^{*}\end{array}$ & $\begin{array}{l}77 \cdot 4 \\
84 \cdot 5 \\
66 \cdot 0\end{array}$ \\
\hline$P V A+T$ & $\begin{array}{l}\text { PVA } \\
\text { PVA }+ \text { T }\end{array}$ & $\begin{array}{c}48 / 79 \\
107 / 150\end{array}$ & $\begin{array}{l}60 \cdot 8 \\
71 \cdot 3\end{array}$ & $\begin{array}{l}(26-100)^{*} \\
(41-94)^{* *}\end{array}$ & $\begin{array}{l}77 \cdot 1 \\
82 \cdot 2\end{array}$ \\
\hline $\mathrm{PVA}+\mathrm{HT}$ & PVA + HT & $39 / 59$ & $66 \cdot 1$ & $(6 \mathrm{I}-71)^{*}$ & $87 \cdot 2$ \\
\hline
\end{tabular}

$\dagger \mathbf{T}=0 \cdot 1$ mM-taurine; $\mathrm{HT}=0 \cdot 1 \mathrm{~mm}$-hypotaurine.

$\ddagger$ These suspensions were not washed.

${ }^{*} P<0.05,{ }^{* *} P<0.01$ compared with PVA washed suspensions.

The possibility that inclusion of taurine in the standard medium with BSA might shorten capacitation was examined by assessing sperm fertility after $30 \mathrm{~min}$ preincubation when control suspensions are generally poorly fertile (e.g. Fig. 1). There was no evidence that sperm fertilizing ability or penetration rate was enhanced in the presence of taurine (results not tabulated).

\section{Series V: acrosome loss}

In washed suspensions after $120 \mathrm{~min}$. Sperm suspensions were prepared in BSA- and PVA-containing media; an aliquant of the latter was washed after $5 \mathrm{~min}$. All suspensions were incubated for $120 \mathrm{~min}$, at which time a sample of the washed group was washed a second time $(\mathrm{N}=3)$. Results (Table 3$)$ indicated that acrosome loss was significantly lower $(P<0.05)$ in the unwashed PVA suspensions than in the BSA control suspensions, but washing promoted loss to levels that did not differ from the controls.

In washed suspensions: time course. Sperm suspensions were prepared in PVA-containing medium and an aliquant was washed at 5 min. During a 120 min incubation, aliquants were 
Table 3. Acrosome loss in mouse sperm suspensions preincubated for 120 min in medium containing BSA or PVA

\begin{tabular}{lcc}
\hline Medium & Treatment & $\begin{array}{c}\text { \% acrosome free-spermatozoa } \\
\text { (mean } \pm \text { s.e.m.) }\end{array}$ \\
\hline BSA & - & $24 \cdot 3 \pm 2 \cdot 7$ \\
PVA & - & $8 \cdot 3 \pm 1 \cdot 5^{*}$ \\
PVA & Wash at $5 \mathrm{~min}$ & $21 \cdot 3 \pm 4 \cdot 4$ \\
PVA & Wash at $5+120 \mathrm{~min}$ & $30 \cdot 7 \pm 2 \cdot 0$ \\
\hline
\end{tabular}

${ }^{*} P<0.05$ compared with BSA control.

Table 4. Acrosome loss in mouse sperm suspensions prepared in PVA-containing medium, washed after $5 \mathrm{~min}$ and then incubated for $120 \mathrm{~min}$

\begin{tabular}{lc}
\hline Incubation $(\mathrm{min})$ & $\begin{array}{c}\text { \% acrosome-free spermatozoa } \\
\text { (mean } \pm \text { s.e.m.) }\end{array}$ \\
\hline 30 & $2 \cdot 7 \pm 0 \cdot 7$ \\
60 & $22 \cdot 7 \pm 5 \cdot 0^{*}$ \\
90 & $23 \cdot 3 \pm 1 \cdot 3^{*}$ \\
120 & $28 \cdot 7 \pm 4 \cdot 4^{* *}$ \\
120 (unwashed) & $7 \cdot 3 \pm 2 \cdot 2$ \\
\hline$* P<0 \cdot 05, * * P<0.01$ compared with 120 min unwashed \\
$\quad$ suspensions.
\end{tabular}

removed from the washed suspension at $30 \mathrm{~min}$ intervals and assessed; the unwashed sample was assessed at 120 min only $(\mathrm{N}=3)$. Results (Table 4) indicated that acrosome loss was minimal ( $3 \%$ ) at $30 \mathrm{~min}$ but had increased significantly to $23 \%$ by $60 \mathrm{~min}$ and thereafter changed little; the unwashed suspensions had fewer than $10 \%$ of acrosome-free spermatozoa after $120 \mathrm{~min}$.

In washed suspensions containing taurine. Sperm suspensions were prepared in PVA-containing medium and washed at $5 \mathrm{~min}$; one aliquant was resuspended in medium containing PVA and $0.1 \mathrm{~mm}$-taurine, a second in PVA only. After $120 \mathrm{~min}$, acrosome loss was assessed $(\mathrm{N}=3)$. There was no increase in the number of acrosome-free spermatozoa in the presence of taurine: PVA, $30.5 \pm 6.5 \%$ (mean \pm s.e.m.); PVA + taurine, $28.3 \pm 6.9 \%$. The possibility that taurine might enhance acrosome loss in unwashed PVA suspensions was examined, but acrosome loss was equally low in both groups (data not presented).

\section{Series VI: motility}

The proportion of motile spermatozoa after 120 min preincubation was estimated in the BSA, PVA, PVA washed and PVA washed + taurine suspensions $(\mathrm{N}=13$ for all except PVA for which $\mathrm{N}=9$ ). The mean percentage motile cells was: BSA, $65 \%$; PVA, $50 \%$; PVA washed, $40 \%$; PVA washed + taurine, $52 \%$. Evaluation of hyperactivated motility suggested that the proportion of spermatozoa exhibiting this pattern of motility in washed suspensions was frequently higher in the presence of taurine, but not consistently so. The differences between suspensions were, in general, quantitative rather than qualitative.

Series VII: analyses of sperm washings

The sperm supernatant $(1 \mathrm{ml}$ per 2 epididymides) removed during washing was subjected to 
acrylamide gel electrophoresis for estimation of albumin content; comparison with BSA standards indicated that $\sim 0.05 \mathrm{mg}$ albumin $/ \mathrm{ml}$ was present in the supernatant.

Amino acid analysis of 5 different supernatant samples revealed that relatively few amino acids were present in a measurable amount. The most prominent components were taurine and hypotaurine, the approximate concentrations in the supernatant being $0.239 \mathrm{~mm}$ and $0.154 \mathrm{~mm}$ respectively. Analysis of the BSA used to prepare control media failed to detect the presence of any amino acids, and negligible amounts were found in the samples prepared from frozen-thawed spermatozoa. Under the conditions used, the elution times were $25.5 \mathrm{~min}$ for taurine and $38-40 \mathrm{~min}$ for hypotaurine. Glycerophosphoethanolamine, which can be a contaminant of taurine (Meizel et al., 1980), eluted at $24.5 \mathrm{~min}$. This is too close to the taurine peak to allow resolution of both substances, if present, but the addition of this compound to a sperm supernatant sample resulted in a peak eluting at $24.5 \mathrm{~min}$. This suggests that glycerophosphoethanolamine is not a major component in the sperm samples, but that taurine is.

\section{Discussion}

These results indicate that washing epididymal mouse spermatozoa free of epididymal fluid immediately after release and then preincubating them in PVA-containing, albumin-free medium results in reduced fertilizing ability when compared with unwashed counterparts preincubated in the same albumin-free medium. However, the reduced fertility of these washed suspensions could be significantly improved in several ways. Washing the suspensions a second time, after $120 \mathrm{~min}$ preincubation, and resuspending in PVA-containing medium was very effective, as was adding BSA $(4 \mathrm{mg} / \mathrm{ml})$ at the end of preincubation. Experiments designed to determine the minimum amount of BSA required to maintain fertilizing ability during preincubation indicated that as little as $0.05-0.1 \mathrm{mg} \mathrm{BSA} / \mathrm{ml}$ would support fertilization levels similar to those achieved in the unwashed control ( $4 \mathrm{mg} \mathrm{BSA} / \mathrm{ml}$ ) suspensions. Furthermore, the continuous presence of low BSA concentrations was more effective in maintaining fertilizing ability than the addition of relatively high concentrations at the end of preincubation (see Figs 1, 2; Table 1). The inclusion of taurine or hypotaurine in PVA-containing medium provided a fourth way to enhance fertilizing ability of the washed PVA suspensions. The positive effect of taurine on sperm function was achieved whether taurine was present during preincubation only, fertilization only or continuously.

Impairment of the ability to undergo the acrosome reaction would reduce sperm fertilizing ability but the data do not indicate that washed spermatozoa were adversely affected in this respect. Acrosome loss was $<10 \%$ after $120 \mathrm{~min}$ preincubation of the unwashed, fertile samples and $>20 \%$ in the washed, poorly fertile samples. This greater loss reflects the removal, by the washing procedure, of a surface-associated inhibitory component which stabilizes sperm membranes and inhibits the acrosome reaction (Fraser, 1984b). Results indicate that this removal leads to the eventual spontaneous loss of acrosomes after about $60 \mathrm{~min}$ (Table 4). The consequence of washing mouse spermatozoa under most conditions is immediate enhancement of fertilizing ability which can be maintained for at least $2 \mathrm{~h}$ (Fig. 1; Fraser, 1984b). It is therefore surprising that the washed PVA suspensions were poorly fertile after either a short or a long preincubation. Albumin or a similar macromolecule is required for the mouse sperm acrosome reaction to occur (Fraser, 1985) and although overt acrosome loss is low in unwashed PVA suspensions, components of the egg-cumulus complex are able to support quite reasonable levels of fertilization (Figs 1 \& 2; Fraser, 1985). Consequently, acrosome reactions would be anticipated following interaction between washed spermatozoa and eggs. When BSA was added to washed suspensions after $30 \mathrm{~min}$ preincubation, they exhibited significantly increased fertility, but when the procedure was repeated after $120 \mathrm{~min}$ the proportion of eggs fertilized had decreased slightly, suggesting that the spermatozoa were affected deleteriously during the albumin-free preincubation. Although earlier studies have suggested that taurine may stimulate the acrosome reaction in hamster spermatozoa 
(Mrsny et al., 1979; Meizel et al., 1980), there is no evidence for a similar involvement in mouse spermatozoa. The inclusion of taurine in unwashed and washed suspensions did not enhance acrosome loss, compared with appropriate amino acid-free suspensions.

Assessment of sperm suspensions after $120 \mathrm{~min}$ preincubation suggested that a major contributory factor to the reduced fertility of washed PVA suspensions was a reduction in the number of motile spermatozoa. The inclusion of taurine enhanced the motility of the suspensions, an observation consistent with the positive effects of taurine on hamster sperm motility (Meizel et al., 1980). It has been suggested that taurine or hypotaurine corresponds to the 'sperm motility factor' which is required for maintenance of hamster sperm motility (see 'Introduction'). Mrsny \& Meizel (1985) have reported that taurine and hypotaurine inhibit hamster sperm $\mathrm{Na}^{+}, \mathrm{K}^{+}$-ATPase activity and suggest that the taurine-associated enhancement of motility may reflect increased availability of ATP and/or increased intracellular $\mathrm{Ca}^{2+}$ resulting from enzyme inhibition. Taurine has been found in the reproductive tract fluids and sperm preparations from several species (Meizel et al., 1980) and the present study has shown the presence of both taurine and hypotaurine in mouse sperm supernatant, presumably contributed primarily by epididymal fluid released into the medium along with the spermatozoa. The evidence therefore suggests that these $\beta$-amino acids can play a role in supporting mouse sperm motility and fertility. This would not appear to be an obligatory requirement, however, since washed spermatozoa supplemented with low concentrations of BSA, which had no detectable taurine or hypotaurine contamination, were motile and more fertile than those supplemented with amino acid only (Tables $1 \& 2$ ). It should be noted that species-related differences in response to taurine and hypotaurine may exist. The range of $\beta$-amino acid concentrations which had positive effects on mouse spermatozoa was $0 \cdot 02-0 \cdot 1 \mathrm{mM}$, with higher values having deleterious effects on sperm fertilizing ability. In contrast, hamster spermatozoa appear to respond positively in terms of motility and fertilizing ability to a wide range of concentrations (0.01-2.0 mu: Mrsny et al., 1979; Leibfried \& Bavister, 1981). Within this range, subsequent studies assessing fertilizing ability have tended to use low concentrations $(0.01 \mathrm{~mm}$; Bavister, 1981), while those assessing acrosome loss, motility and biochemical parameters have utilized considerably higher concentrations (0.5 mM: Meizel et al., 1980; Mrsny \& Meizel, 1985).

This study has shown that removal of epididymal fluid components from mouse sperm suspensions immediately upon release from the epididymis has deleterious consequences for maintenance of fertility. Among the constituents, present in the suspension and removed by washing, are albumin, albeit at a low concentration of $\sim 0.05 \mathrm{mg} / \mathrm{ml}$ supernatant, taurine and hypotaurine. The reintroduction of albumin at a similarly low concentration is sufficient to protect the spermatozoa and maintain full fertilizing ability. The enhancement of fertilizing ability to somewhat lower levels, by introduction of albumin or taurine or by further washing of the spermatozoa after an extended preincubation, indicates that these cells can be 'rescued' by apparently diverse methods. Whether a mechanism of action common to all is involved is conjectural at present. The fact that washing a second time improves fertility, even in the absence of any added albumin, suggests the accumulation of some inhibitory factor(s) which can be removed by the washing procedure. Studies on rabbit spermatozoa have indicated that taurine, hypotaurine and albumin can inhibit lipid peroxidation which would otherwise cause motility loss (Alvarez \& Storey, 1982, 1983). Perhaps a feature common to these different approaches for enhancing fertilizing ability is the removal of potentially damaging molecules from the suspension. Clearly the greatest protection is provided by the continuous presence of albumin; the reduced response to albumin after an extended incubation suggests that permanent damage to some of the sperm population has occurred. There is no evidence to indicate an obligatory taurine requirement for maintenance of motility or support of the acrosome reaction in mouse spermatozoa.

This work was supported in part by a grant from the AFRC. I thank N. Harper for the acrylamide gel analyses and T. Crutcher for the amino acid analyses. 


\section{References}

Alvarez, J.G. \& Storey, B.T. (1982) Spontaneous lipid peroxidation in rabbit epididymal spermatozoa: its effect on sperm motility. Biol. Reprod. 27, 1102-1108.

Alvarez, J.G. \& Storey, B.T. (1983) Taurine, hypotaurine, epinephrine and albumin inhibit lipid peroxidation in rabbit spermatozoa and protect against loss of motility. Biol. Reprod. 29, 548-555.

Bavister, B.D. (1981) Substitution of a synthetic polymer for protein in a mammalian gamete culture system. $J$. exp. Zool. 217, 45-51.

Bedford, J.M. (1970) Sperm capacitation and fertilization in mammals. Biol. Reprod., Suppl. 2, 128-158.

Fraser, L.R. (1983) Potassium ions modulate expression of mouse sperm fertilizing ability, acrosome reaction and hyperactivated motility in vitro. J. Reprod. Fert. 69, 539-553.

Fraser, L.R. (1984a) Mechanisms controlling mammalian fertilization. In Oxford Reviews of Reproductive Biology, Vol 6, pp. 174-225. Ed. J. R. Clarke. Oxford University Press.

Fraser, L.R. (1984b) Mouse sperm capacitation in vitro involves loss of a surface-associated inhibitory component. J. Reprod. Fert. 72, 373-384.

Fraser, L.R. (1985) Albumin is required to support the acrosome reaction but not capacitation in mouse spermatozoa in vitro. J. Reprod. Fert. 74, 185 196

Harrison, R.A.P., Dott, H.M. \& Foster, G.C. (1982) Bovine serum albumin, sperm motility, and the 'dilution effect'. J. exp. Zool. 222, 81-88.
Leibfried, M.L. \& Bavister, B.D. (1981) The effects of taurine and hypotaurine on in vitro fertilization in the golden hamster. Gamete Res. 4, 57-63.

Lui, C.W. \& Meizel, S. (1977) Biochemical studies of the in vitro acrosome reaction inducing activity of bovine serum albumin. Differentiation 9, 59-66.

Meizel, S., Lui, C.W., Working, P.K. \& Mrsny, R.J. (1980) Taurine and hypotaurine: their effects on motility, capacitation and the acrosome reaction of hamster sperm in vitro and their presence in sperm and reproductive tract fluids of several mammals. Develop., Growth and Differ. 22, 483-494.

Mrsny, R.J. \& Meizel, S. (1985) Inhibition of hamster sperm $\mathrm{Na}^{+}, \mathrm{K}^{+}$-ATPase activity by taurine and hypotaurine. Life Sci. 36, 271-275.

Mrsny, R.J., Waxman, L. \& Meizel, S. (1979) Taurine maintains and stimulates motility of hamster sperm during capacitation in vitro. J. exp. Zool. 210, $123-128$

Snedecor, G. \& Cochran, W. (1967) Statistical Methods, 6 th edn. Iowa State University Press, Ames.

Suter, D., Chow, P.Y.W. \& Martin, I.C.A. (1979) Maintenance of motility in human spermatozoa by energy derived through oxidative phosphorylation and addition of albumin. Biol. Reprod. 20, 505-510.

Received 27 September 1985 